\title{
Open book decompositions versus prime factorizations of closed, oriented 3-manifolds
}

\author{
PAOLO GhigGini \\ PAOLO LISCA
}

\begin{abstract}
Let $M$ be a closed, oriented, connected 3 -manifold and $(B, \pi)$ an open book decomposition on $M$ with page $\Sigma$ and monodromy $\varphi$. It is easy to see that the first Betti number of $\Sigma$ is bounded below by the number of $S^{2} \times S^{1}$-factors in the prime factorization of $M$. Our main result is that equality is realized if and only if $\varphi$ is trivial and $M$ is a connected sum of copies of $S^{2} \times S^{1}$. We also give some applications of our main result, such as a new proof of the fact that if the closure of a braid with $n$ strands is the unlink with $n$ components then the braid is trivial.
\end{abstract}

$57 \mathrm{~N} 10 ; 57 \mathrm{M} 25$

\section{Introduction}

An abstract open book is a pair $(\Sigma, \varphi)$, where $\Sigma$ is a connected, oriented surface with $\partial \Sigma \neq \varnothing$ and the monodromy $\varphi$ is an element of the group $\operatorname{Diff}^{+}(\Sigma, \partial \Sigma)$ of orientationpreserving diffeomorphisms of $\Sigma$ which restrict to the identity on a neighborhood of the boundary. We say that the monodromy $\varphi$ is trivial if it is isotopic to the identity of $\Sigma$ via diffeomorphisms which fix $\partial \Sigma$ pointwise. Let $N_{\varphi}$ denote the mapping torus

$$
N_{\varphi}=\Sigma \times[0,1] /(p, 1) \sim(\varphi(p), 0) .
$$

To the open book $(\Sigma, \varphi)$ one can associate a closed, oriented, connected 3-manifold $M_{(\Sigma, \varphi)}$ by using the natural identification of $\partial N_{\varphi}=\partial \Sigma \times S^{1}$ with the boundary of $\partial \Sigma \times D^{2}$ :

$$
M_{(\Sigma, \varphi)}:=N_{\varphi} \cup_{\partial} \partial \Sigma \times D^{2} .
$$

The link $B:=\partial \Sigma \times\{0\} \subset M_{(\Sigma, \varphi)}$ is fibered, with fibration $\pi: M_{(\Sigma, \varphi)} \backslash B \rightarrow S^{1}$ given by the obvious extension of the natural projection

$$
N_{\varphi}=\Sigma \times[0,1] /(p, 1) \sim(\varphi(p), 0) \rightarrow S^{1}=[0,1] / 1 \sim 0
$$


and monodromy equal to $\varphi$. In other words, the pair $(B, \pi)$ is an open book decomposition of $M=M_{(\Sigma, \varphi)}$ with binding $B$, pages $\Sigma_{\theta}:=\overline{\pi^{-1}(\theta)}, \theta \in S^{1}$, and monodromy $\varphi$. We will always identify $N_{\varphi}$ with the complement of a tubular neighborhood of $B$ in $M$.

If $(B, \pi)$ is an open book decomposition of $M$ with page $\Sigma$, it is easy to see that $M$ has a Heegaard splitting of genus $b_{1}(\Sigma)$. Since $M$ is obtained from each handlebody of the splitting by attaching 2-disks and 3-balls, this immediately implies the inequality

$$
b_{1}(M) \leq b_{1}(\Sigma) .
$$

We will provide a refinement of Inequality (1) in Proposition 2.2.

The following theorem is our main result. Its proof is based on well-known results due to Reidemeister [14], Singer [15] and Haken [8] (see Section 3). Recall that each closed, oriented, connected 3-manifold $M$ has a prime factorization, unique up to order of the factors, of the form

$$
M=M_{1} \# \cdots \# M_{h} \# S^{2} \times S^{1} \# \stackrel{(k)}{*} \# S^{2} \times S^{1},
$$

where each $M_{i}$ is irreducible (see eg [10]).

Theorem 1.1 Let $(B, \pi)$ be an open book decomposition of a closed, oriented, connected 3-manifold $M$ with page $\Sigma$ and monodromy $\varphi$. Then $b_{1}(\Sigma)$ is equal to the number of $S^{2} \times S^{1}$-factors in the prime factorization of $M$ if and only if $\varphi$ is trivial and $M$ is a connected sum of copies of $S^{2} \times S^{1}$.

Theorem 1.1 immediately implies the following corollary, which is also proved in Ni [12, Proof of Theorem 1.3] and Grigsby and Wehrli [7, Theorem 2] using the fact that finitely generated free groups are not isomorphic to any of their nontrivial quotients.

Corollary 1.2 Any open book decomposition of $\#^{k} S^{2} \times S^{1}$ whose page $\Sigma$ satisfies $b_{1}(\Sigma)=k$ must have trivial monodromy.

Corollary 1.2 implies Corollary 1.3, which was obtained previously by Cochran [3] using the fact that finitely generated free groups are not isomorphic to any of their nontrivial quotients, and by Birman and Menasco [2] as an application of their braid foliation techniques. Grigsby and Wehrli [7] gave another proof of Corollary 1.3 using Khovanov homology.

Corollary 1.3 Let $b \in B_{n}$ be a braid on $n$ strands such that its closure $\hat{b}$ is the trivial link $U_{n}$ with $n$ components. Then $b$ is the identity. 
Proof Put $\hat{b}$ in braid form with respect to the binding of the trivial open book decomposition of $S^{3}$ and consider the two-fold branched cover $\Sigma(\hat{b})$ along $\hat{b}$. Then

$$
\Sigma(\hat{b})=\Sigma\left(U_{n}\right)=\stackrel{n-1}{\#} S^{2} \times S^{1} .
$$

Pulling back the trivial open book of $S^{3}$ to $\Sigma(\widehat{b})$ we obtain an open book decomposition of $\#^{n-1} S^{2} \times S^{1}$, whose page is a surface $\Sigma$ with $b_{1}(\Sigma)=n-1$, which we view as a 2 -fold branched cover of the disk with $n$ branch points. Under the identification of $B_{n}$ with the subgroup of the mapping class group of $\Sigma$ given by the elements commuting with the covering involution [1], the monodromy of the open book is equal to $b$. By Corollary 1.2, the braid $b$ must be the identity in $B_{n}$.

Let $\Sigma$ and $\Sigma^{\prime}$ be two orientable surfaces. By performing a boundary connected sum between them we obtain a surface $\Sigma \curvearrowleft \Sigma^{\prime}$. If $\varphi$ is a diffeomorphism of $\Sigma, \psi$ is a diffeomorphism of $\Sigma^{\prime}$ and both $\varphi$ and $\psi$ are the identity on a neighborhood of the boundary, we can form a diffeomorphism $\varphi \downarrow \psi$ of $\Sigma \curvearrowleft \Sigma^{\prime}$. This geometric operation yields a homomorphism

$$
\Gamma_{\Sigma} \times \Gamma_{\Sigma^{\prime}} \rightarrow \Gamma_{\Sigma \natural \Sigma^{\prime}}
$$

which we will call boundary connected sum homomorphism. A combination of Inequality (1) with Corollary 1.2 yields the following Corollary 1.4, which can also be proved by applying [13, Corollary 4.2 (iii)].

Corollary 1.4 Let $\Gamma_{\Sigma}$ be the mapping class group of the orientable surface $\Sigma$. Then the boundary connected sum homomorphism

$$
\Gamma_{\Sigma} \times \Gamma_{\Sigma^{\prime}} \rightarrow \Gamma_{\Sigma \llbracket \Sigma^{\prime}}
$$

is injective.

Proof Under the map $(\Sigma, \varphi) \rightarrow M_{(\Sigma, \varphi)}$ described above, boundary connected sum of abstract open books corresponds to connected sum of 3-manifolds:

$$
M_{\left(\Sigma \natural \Sigma^{\prime}, \varphi \natural \psi\right)}=M_{(\Sigma, \varphi)} \# M_{\left(\Sigma^{\prime}, \psi\right)} .
$$

Observe that $b_{1}\left(\Sigma \curvearrowleft \Sigma^{\prime}\right)=b_{1}(\Sigma)+b_{1}\left(\Sigma^{\prime}\right)$. Therefore, if $\varphi \downarrow \psi$ is isotopic to the identity relative to the boundary then $M_{\left(\Sigma \natural \Sigma^{\prime}, \varphi \natural \psi\right)}$ is diffeomorphic to $\#^{b_{1}(\Sigma)+b_{1}\left(\Sigma^{\prime}\right)} S^{2} \times S^{1}$. The uniqueness of the prime factorization for 3-manifolds [10] implies that

$$
M_{(\Sigma, \varphi)}=\stackrel{k}{\#} S^{2} \times S^{1} \quad \text { and } \quad M_{\left(\Sigma^{\prime}, \psi\right)}=\stackrel{l}{\#} S^{2} \times S^{1}
$$


for some nonnegative integers $k, l$ such that $k+l=b_{1}(\Sigma)+b_{1}\left(\Sigma^{\prime}\right)$. By Inequality (1) we have $k \leq b_{1}(\Sigma)$ and $l \leq b_{1}\left(\Sigma^{\prime}\right)$, which forces $k=b_{1}(\Sigma)$ and $l=b_{1}\left(\Sigma^{\prime}\right)$ as the only possibility. Corollary 1.2 implies that $\varphi$ and $\psi$ are isotopic to the identity.

The rest of the paper is organized as follows. In Section 2 we recall two well-known results independent of Theorem 1.1, ie Propositions 2.1 and 2.2. Proposition 2.1 shows that any embedded 2-sphere disjoint from the binding of an open book decomposition bounds an embedded ball. Proposition 2.2 is a refinement of Inequality (1) and can be viewed as saying that the homology of a closed, oriented, connected 3-manifold $M$ puts homological constrains on the monodromy of any open book decomposition of $M$. In Section 3 we prove Theorem 1.1.

Acknowledgements The authors wish to thank the anonymous referees for valuable comments. The present work is part of the authors' activities within CAST, a Research Network Program of the European Science Foundation. The first author was partially supported by the ERC grant "Geodycon". The second author was partially supported by the PRIN-MIUR research project 2010-2011 "Varietà reali e complesse: geometria, topologia e analisi armonica".

\section{Nonseparating 2-spheres and refinement of Inequality (1)}

Given a closed, oriented, connected 3-manifold $M$ endowed with an open book decomposition $(B, \pi)$ and having a prime factorization as in (2), one of the first questions one could ask is how a nonseparating 2 -sphere $S$ in $M$ can be positioned with respect to the binding $B$. Since $B$ is homologically trivial in $M$, the following proposition implies that, possibly after a small isotopy, each such $S$ must intersect $B$ transversally at least twice.

Proposition 2.1 Let $(B, \pi)$ be an open book decomposition with page $\Sigma$ and monodromy $\varphi$ of a closed, oriented, connected 3-manifold $M$. Then each embedded 2-sphere $S \subset M \backslash B$ bounds an embedded ball in $M \backslash B$ and, in particular, is homologically trivial in $M$.

Proof Recall that $M=N_{\varphi} \cup V$, where $V$ is a tubular neighborhood of the binding. Up to an isotopy of $S$, we can assume $S \subset N_{\varphi}$. The universal cover of $N_{\varphi}$ is homeomorphic to $\mathbb{R}^{3}$ and from this the triviality of $[S]$ in $H_{2}(M \backslash B)$, and therefore in $H_{2}(M)$, follows immediately.

In order to prove that $S$ bounds a ball in $M \backslash B$ we need to use some basic results in three-dimensional topology. In fact $\mathbb{R}^{3}$ is irreducible [9, Theorem 1.1] and this 
implies [9, Proposition 1.6] that $N_{\varphi}$ is also irreducible, therefore $S$ bounds an embedded ball in $N_{\varphi}$.

We now establish a result which refines Inequality (1). Proposition 2.2 below can be viewed as saying that the homology of a closed, oriented, connected 3-manifold $M$ puts homological constraints on the monodromy of any open book decomposition of $M$.

For the rest of this section all homology groups will be taken with coefficients in the field $\mathbb{Q}$ of rational numbers unless specified otherwise. Let $H_{1}(\Sigma, \partial \Sigma)^{\varphi}$ denote the subspace of $H_{1}(\Sigma, \partial \Sigma)$ consisting of the elements fixed by the map

$$
\varphi_{*}: H_{1}(\Sigma, \partial \Sigma) \rightarrow H_{1}(\Sigma, \partial \Sigma)
$$

induced by the monodromy $\varphi: \Sigma \rightarrow \Sigma$.

Proposition 2.2 Let $(B, \pi)$ be an open book decomposition with page $\Sigma$ and monodromy $\varphi$ of a closed, oriented, connected 3-manifold $M$. Then

$$
b_{1}(M)=\operatorname{dim}_{\mathbb{Q}} H_{1}(\Sigma, \partial \Sigma)^{\varphi} .
$$

More precisely, there is an isomorphism $H_{2}(M) \cong H_{1}(\Sigma, \partial \Sigma)^{\varphi}$ induced by a welldefined map $H_{2}(M ; \mathbb{Z}) \rightarrow H_{1}(\Sigma, \partial \Sigma ; \mathbb{Z})^{\varphi}$ given by $\alpha \mapsto[F \cap \Sigma]$, where $F \subset M$ is any closed, oriented and properly embedded surface which represents $\alpha$ and intersects the page $\Sigma \times\{0\}$ transversally.

Proof We can view $N_{\varphi}$ as the union of $\Sigma \times\left[0, \frac{1}{2}\right]$ and $\Sigma \times\left[\frac{1}{2}, 1\right]$ with $(x, 1)$ identified to $(\varphi(x), 0)$. Using the fact that $\Sigma$ times an interval is homotopically equivalent to $\Sigma$, the (relative) Mayer-Vietoris sequence for this splitting gives the exact sequence

$$
H_{2}(\Sigma, \partial \Sigma)^{2} \stackrel{f_{1}}{\longrightarrow} H_{2}\left(N_{\varphi}, \partial N_{\varphi}\right) \stackrel{f_{2}}{\longrightarrow} H_{1}(\Sigma, \partial \Sigma)^{2} \stackrel{f_{3}}{\longrightarrow} H_{1}(\Sigma, \partial \Sigma)^{2} .
$$

The map $f_{3}$ is given by the matrix

$$
\left(\begin{array}{ll}
\text { Id } & \text { Id } \\
\varphi_{*} & \text { Id }
\end{array}\right) \in M_{2}\left(\operatorname{End}\left(H_{1}(\Sigma, \partial \Sigma)\right)\right)
$$

This immediately implies that the image of $f_{2}$ is isomorphic to $H_{1}(\Sigma, \partial \Sigma)^{\varphi}$.

Recall the decomposition $M=N_{\varphi} \cup V$, where $V$ is a tubular neighborhood of the binding. Since $H_{2}(V)=\{0\}$, the homology exact sequence for the pair $(M, V)$ implies that the map $g: H_{2}(M) \rightarrow H_{2}(M, V)$ induced by the inclusion map is injective. On the other hand, by excision the inclusion $N_{\varphi} \subset M$ induces an isomorphism

$$
\psi: H_{2}\left(N_{\varphi}, \partial N_{\varphi}\right) \stackrel{\cong}{\longrightarrow} H_{2}(M, V) .
$$


Moreover, it is easy to see that the image of the map $\psi \circ f_{1}$ maps injectively to $H_{1}(V)$ under the next map $\delta: H_{2}(M, V) \rightarrow H_{1}(V)$ in the exact sequence of the pair, while the image of $g$ maps trivially. This shows that the images of $f_{1}$ and of $\psi^{-1} \circ g$ have trivial intersection. Therefore the composition $f_{2} \circ \psi^{-1} \circ g$ sends $H_{2}(M)$ injectively into the image of the map $f_{2}$, which, as we have just shown, is isomorphic to $H_{2}(\Sigma, \partial \Sigma)^{\varphi}$.

We claim that $f_{2} \circ \psi^{-1} \circ g$ sends $H_{2}(M)$ also surjectively onto the image of $f_{2}$. In order to verify this, we argue by induction. Assume first that $\partial \Sigma$ is connected. In this situation the map $\delta \circ \psi \circ f_{1}$ is clearly surjective. Therefore, if $x \in H_{2}\left(N_{\varphi}, \partial N_{\varphi}\right)$ with $f_{2}(x) \neq 0$, there exists $y \in H_{2}(\Sigma, \partial \Sigma)^{2}$ with $\delta \circ \psi \circ f_{1}(y)=\delta \circ \psi(x)$. It follows that setting $x^{\prime}=x-f_{1}(y)$ we have $f_{2}\left(x^{\prime}\right)=f_{2}(x)$ and $\delta \circ \psi\left(x^{\prime}\right)=0$; therefore $x^{\prime}$ is in the image of $\psi^{-1} \circ g$, and the claim is proved when $\partial \Sigma$ is connected.

Now assume $\partial \Sigma$ is disconnected and denote by $|\partial \Sigma|$ the number of its connected components. By the inductive hypothesis we assume that the claim holds for open books with $|\partial \Sigma|-1$ binding components. Let $(\hat{\Sigma}, \hat{\varphi})$ be another abstract open book, constructed as follows. The connected, oriented surface $\hat{\Sigma}$ is obtained by attaching a 2 -dimensional 1 -handle $h$ to $\partial \Sigma$ so that $|\partial \hat{\Sigma}|=|\partial \Sigma|-1$, while $\hat{\varphi}$ is defined by first extending $\varphi$ as the identity over $h$, and then composing with a (positive or negative) Dehn twist along a simple closed curve in $\widehat{\Sigma}$ which intersects the cocore $c$ of $h$ transversely once. It is a well-known fact that the open book decomposition $(\widehat{B}, \hat{\pi})$ associated to $(\hat{\Sigma}, \hat{\varphi})$ is obtained from the open book decomposition $(B, \pi)$ associated to $(\Sigma, \varphi)$ by plumbing with a Hopf band, and that $M_{(\widehat{\Sigma}, \hat{\varphi})}$ is diffeomorphic to $M$ (see [6]). We can choose a basis $\left[c_{1}\right], \ldots,\left[c_{b_{1}(\Sigma)}\right]$ of $H_{1}(\Sigma, \partial \Sigma)$ such that each $c_{i} \subset \Sigma$ is a properly embedded arc disjoint from $\gamma \cap \Sigma$, and so that, viewing the classes $\left[c_{i}\right]$ in $H_{1}(\hat{\Sigma}, \partial \hat{\Sigma})$, when we add $[c]$ we obtain a basis of $H_{1}(\hat{\Sigma}, \partial \hat{\Sigma})$. Using this basis one can easily check that the natural inclusion map $H_{1}(\Sigma, \partial \Sigma) \rightarrow H_{1}(\hat{\Sigma}, \partial \hat{\Sigma})$ restricts to an isomorphism

$$
H_{1}(\Sigma, \partial \Sigma)^{\varphi} \cong H_{1}(\widehat{\Sigma}, \partial \widehat{\Sigma})^{\hat{\varphi}}
$$

Since $|\partial \hat{\Sigma}|=|\partial \Sigma|-1$, by the inductive assumption we have

$$
b_{1}(M)=\operatorname{dim}_{\mathbb{Q}} H_{1}(\hat{\Sigma}, \partial \hat{\Sigma})^{\hat{\varphi}} .
$$

This proves the claim in full generality. Finally, observe that the maps $f_{2}$ and $f_{2} \circ \psi^{-1} \circ g$ are well-defined over the integers. If we represent homology classes in $H_{2}\left(N_{\varphi}, \partial N_{\varphi} ; \mathbb{Z}\right)$ and $H_{2}(M ; \mathbb{Z})$ by oriented, properly embedded surfaces intersecting the page $\Sigma \times\{0\}$ transversally and we follow the construction of the connecting homomorphism, we see that the maps $f_{2}$ and $f_{2} \circ \psi^{-1} \circ g$ are both realized geometrically by intersecting with $\Sigma \times\{0\}$. This concludes the proof. 


\section{The proof of Theorem 1.1}

We start by recalling a basic result of Reidemeister and Singer about collections of compressing disks in a handlebody. We refer to [11] for a modern presentation of this material. Let $H_{g}$ be a 3-dimensional handlebody of genus $g$. A properly embedded disk $D \subset H_{g}$ is essential if $\partial D$ does not bound a disk in $\partial H_{g}$.

Definition 3.1 A collection $\left\{D_{1}, \ldots, D_{g}\right\} \subset H_{g}$ of $g$ properly embedded, pairwise disjoint essential disks is a minimal system of disks for $H_{g}$ if the complement of a regular neighborhood of $\bigcup_{i} D_{i}$ in $H_{g}$ is homeomorphic to a 3-dimensional ball.

Let $D_{1}, D_{2} \subset H$ be properly embedded, essential disks in the handlebody $H_{g}$. Let $a \subset \partial H$ be an embedded arc with one endpoint on $\partial D_{1}$ and the other endpoint on $\partial D_{2}$. Let $N$ be the closure of a regular neighborhood of $D_{1} \cup D_{2} \cup a$ in $H$. Then $N$ is homeomorphic to a closed 3-ball, and it intersects $\partial H_{g}$ in a subset of $\partial N$ homeomorphic to a three-punctured 2-sphere. The complement $\partial N \backslash \partial H_{g}$ of this subset consists of the disjoint union of three disks, two of which are isotopic to $D_{1}$ and $D_{2}$ respectively, and the third one is denoted by $D_{1} *_{a} D_{2}$. See Figure 1.

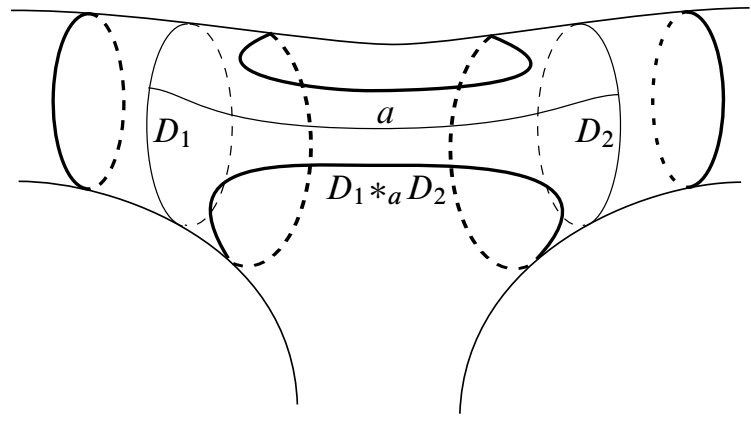

Figure 1: A disk slide

Let $\boldsymbol{D}=\left\{D_{1}, \ldots, D_{g}\right\}$ be a minimal system of disks for a handlebody $H_{g}, a \subset \partial H_{g}$ an embedded arc with one endpoint on $\partial D_{i}$, the other endpoint on $\partial D_{j}$, with $i \neq j$, and the interior of $a$ disjoint from $\bigcup_{i} \partial D_{i}$. Then, removing either $D_{i}$ or $D_{j}$ from $\boldsymbol{D}$ and adding $D_{i} *_{a} D_{j}$ yields a new minimal system of disks $\boldsymbol{D}^{\prime}$ for $H_{g}$, well-defined up to isotopy [11, Corollary 2.11]. In this situation we say that $\boldsymbol{D}^{\prime}$ is obtained from $\boldsymbol{D}$ by a disk slide.

Definition 3.2 Two minimal systems of disks for $H_{g}$ are slide equivalent if they are connected by a finite sequence $\boldsymbol{D}_{1}, \ldots, \boldsymbol{D}_{m}$ such that $\boldsymbol{D}_{i+1}$ is obtained from $\boldsymbol{D}_{i}$ by a disk slide for each $i$. 
To prove Theorem 1.1 we need the following result (see [11, Theorem 2.13] for a modern exposition).

Theorem 3.3 [14; 15] Any two minimal systems of disks for a handlebody are slide equivalent.

We can now start the formal proof of Theorem 1.1. The first step is to normalize the position of certain nonseparating $2-$ spheres with respect to a Heegaard splitting. This will be done in the following lemma.

Lemma 3.4 Let $M=H \cup H^{\prime}$ be a Heegaard splitting of a 3-manifold $M$ which admits a prime factorization

$$
M=M_{1} \# \cdots \# M_{h} \# S^{2} \times S^{1} \# \stackrel{(k)}{\cdots} \# S^{2} \times S^{1}
$$

with $b_{1}(M)=k$. Then there are pairwise disjoint, embedded 2-spheres $S_{1}, \ldots, S_{k}$ in $M$ such that each $S_{i}$ intersects the Heegaard surface $\partial H$ in a single circle $C_{i}$. Moreover, after choosing an orientation of each $S_{i}$, the corresponding 2-homology classes $\left[S_{i}\right]$ generate $\mathrm{H}_{2}(M ; \mathbb{Q})$.

Proof Suppose that $M^{\prime}=M_{1} \# \cdots \# M_{h}$, where each $M_{i}$ is irreducible. By definition any embedded 2-sphere $S \subset M_{i}$ bounds a 3-ball. Therefore, if we denote by $S_{1}^{\prime}, \ldots, S_{h-1}^{\prime} \subset M^{\prime}$ the separating spheres along which the connected sums are performed and $S_{h}^{\prime} \subset M^{\prime}$ is any smoothly embedded 2-sphere disjoint from $S_{1}^{\prime}, \ldots, S_{h-1}^{\prime}$, then the closure of some component of $M^{\prime} \backslash \bigcup_{i=1}^{h} S_{i}^{\prime}$ is a punctured 3-ball.

In the terminology of Haken [8], a collection of pairwise disjoint, embedded 2-spheres with such a property is called a complete system of spheres. Thus, the collection $S_{1}^{\prime}, \ldots, S_{h-1}^{\prime}$ is a complete system of spheres for $M^{\prime}$. If we view each sphere $S_{i}^{\prime}$ as contained in $M$ and denote by $S_{h-1+i}^{\prime} \subset M$, for $i=1, \ldots, k$, the embedded 2-sphere corresponding to $S^{2} \times\{1\}$ in the $i^{\text {th }} S^{2} \times S^{1}$-factor of the factorization (3), the whole collection $S_{1}^{\prime}, \ldots, S_{h-1}^{\prime}, S_{h}^{\prime}, \ldots, S_{h-1+k}^{\prime}$ is a complete system of spheres for $M$.

Observe that, since $b_{1}(M)=k, b_{1}\left(M^{\prime}\right)=0$. Then, after choosing orientations, the homology classes $\left[S_{h-1+i}^{\prime}\right] \in H_{2}(M ; \mathbb{Q})$ generate $H_{2}(M ; \mathbb{Q})$ as a $\mathbb{Q}$-vector space, and a fortiori the same is true for the classes $\left[S_{1}^{\prime}\right], \ldots,\left[S_{h-1+k}^{\prime}\right]$.

Now, according to the lemma on page 84 of [8], the system of spheres $S_{1}^{\prime}, \ldots, S_{h-1+k}^{\prime}$ may be transformed by a finite sequence of isotopies and " $\rho$-operations" (see [8] for the definition) into a collection of pairwise disjoint, incompressible 2-spheres $S_{1}, \ldots, S_{t}$, $t \geq h-1+k$, such that each $S_{i}$ intersects the Heegaard surface $\partial H$ in a single 
circle $C_{i}=S_{i} \cap \partial H$, and moreover the classes $\left[S_{i}\right]$ still generate $H_{2}(M ; \mathbb{Q})$. Since $\operatorname{dim}_{\mathbb{Q}} H_{2}(M ; \mathbb{Q})=k$, up to renaming the spheres we may assume that $\left[S_{1}\right], \ldots,\left[S_{k}\right]$ are generators of $\mathrm{H}_{2}(M ; \mathbb{Q})$. This finishes the proof of the lemma.

Proof of Theorem 1.1 Let $(B, \pi)$ be an open book decomposition of a closed, oriented, connected 3-manifold $M$ with page $\Sigma$ and monodromy $\varphi$. If $\varphi$ is trivial then it is easy to check that $M$ is homeomorphic to the connected sum of $b_{1}(\Sigma)$ copies of $S^{2} \times S^{1}$. This proves one direction of the statement. For the other direction, suppose that $M$ factorizes as in (2). In view of Proposition 2.2 or Inequality (1) we have

$$
b_{1}(\Sigma) \geq b_{1}(M) \geq k .
$$

If $b_{1}(\Sigma)=k$, the above inequality implies $b_{1}(M)=k$ and therefore if we set

$$
M^{\prime}:=M_{1} \# \cdots \# M_{h}
$$

we have $b_{1}\left(M^{\prime}\right)=0$.

Denote by $H_{b_{1}(\Sigma)} \subset M$ the handlebody of genus $b_{1}(\Sigma)$ consisting of a regular neighborhood of $\Sigma$ in $M$. Since $\Sigma$ is the fiber of a fibration, the closure of the complement $M \backslash H_{b_{1}(\Sigma)}$ is a handlebody as well, which we denote by $H_{b_{1}(\Sigma)}^{\prime}$. It follows that $M$ admits the Heegaard splitting

$$
M=H_{b_{1}(\Sigma)} \cup H_{b_{1}(\Sigma)}^{\prime} .
$$

By Lemma 3.4 there are pairwise disjoint embedded spheres $S_{1}, \ldots, S_{k} \subset M$ which generate $H_{2}(M ; \mathbb{Q})$ and such that each $S_{i}$ intersects the Heegaard surface $\partial H_{b_{1}(\Sigma)}$ in a single circle $C_{i}$.

Observe that each circle $C_{i}$ bounds the disk $D_{i}=S_{i} \cap H_{b_{1}(\Sigma)}$ inside $H_{b_{1}(\Sigma)}$ and the disk $S_{i} \cap H_{b_{1}(\Sigma)}^{\prime}$ inside $H_{b_{1}(\Sigma)}^{\prime}$. Since the map

$$
H_{2}(M ; \mathbb{Q}) \rightarrow H_{1}\left(\partial H_{b_{1}}(\Sigma) ; \mathbb{Q}\right)
$$

appearing in the Mayer-Vietoris sequence associated with the decomposition (4) is injective, after choosing orientations we see that the induced homology classes $\left[C_{i}\right]$ generate a half-dimensional subspace of $H_{1}\left(\partial H_{b_{1}(\Sigma)} ; \mathbb{Q}\right)$ which is Lagrangian for the intersection form on $H_{1}\left(\partial H_{b_{1}(\Sigma)} ; \mathbb{Q}\right)$ because the $C_{i}$ are pairwise disjoint.

We now claim that the $D_{i}$ are a minimal system of compressing disks for $H_{b_{1}(\Sigma)}$. To see this we can argue by induction on $b_{1}(\Sigma)$. If $b_{1}(\Sigma)=0$ there is nothing to prove, so we may assume $b_{1}(\Sigma)>0$. Let $N$ be an open regular neighborhood of $D_{1}$.

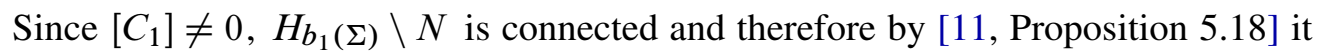
is a handlebody. Moreover, the remaining homology classes $\left[C_{i}\right], i \geq 2$, generate a 
Lagrangian subspace in the first homology group of the boundary of $H_{b_{1}(\Sigma)} \backslash N$. By the inductive assumption the disks $D_{i}$ for $i \geq 2$ are a minimal system of compressing disks for $H_{b_{1}(\Sigma)} \backslash N$, which proves the claim.

Recall that, by construction, the curves $C_{i}=\partial D_{i}$ bound compressing disks in $H_{b_{1}(\Sigma)}^{\prime}$. Arguing as for $H_{b_{1}(\Sigma)}$ shows that such disks constitute a minimal system for $H_{b_{1}(\Sigma)}^{\prime}$. Thus, surgering $M$ along the spheres $S_{1}, \ldots, S_{k}$ yields a 3 -manifold having a genus- 0 Heegaard splitting, ie $S^{3}$. This implies that $M$ is a connected sum of $k$ copies of $S^{2} \times S^{1}$, and we are left to show that the monodromy $\varphi$ is trivial.

Now we choose a system of arcs for $\Sigma$, ie a collection of properly embedded, pairwise disjoint oriented $\operatorname{arcs} a_{1}, \ldots, a_{b_{1}(\Sigma)} \subset \Sigma$ whose associated homology classes $\left[a_{i}\right] \in$ $H_{1}(\Sigma, \partial \Sigma ; \mathbb{Q})$ generate the $\mathbb{Q}$-vector space $H_{1}(\Sigma, \partial \Sigma ; \mathbb{Q})$. Then, after fixing an identification $H_{b_{1}(\Sigma)}=\Sigma \times I$, the disks $a_{i} \times I \subset \Sigma \times I$ yield another minimal system of disks $\left\{D_{i}^{\prime}\right\}_{i=1}^{g}$ for $H_{b_{1}(\Sigma)}$. Thus, according to Theorem 3.3, the system $\left\{D_{i}\right\}_{i=1}^{g}$ is slide equivalent to the system $\left\{D_{i}^{\prime}\right\}_{i=1}^{g}$. But recall that, by construction, each curve $C_{i}=\partial D_{i}$ bounds a compressing disk in $H_{b_{1}(\Sigma)}^{\prime}$, and a moment's reflection shows that any disk slide among the $D_{i}$ gives rise to a disk $D_{i} *_{a} D_{j}$ whose boundary also bounds a compressing disk in $H_{b_{1}(\Sigma)}^{\prime}$. By induction we conclude that any minimal system of disks $\left\{\widetilde{D}_{i}\right\}_{i=1}^{g}$ obtained from $\left\{D_{i}\right\}_{i=1}^{g}$ by a finite sequence of isotopies and disk slides still has the property that each curve $\partial \widetilde{D}_{i}$ bounds a compressing disk in $H_{b_{1}}^{\prime}(\Sigma)$.

In particular, this conclusion applies to the system $\left\{D_{i}^{\prime}\right\}_{i=1}^{g}$, showing that each of the circles $\partial D_{i}^{\prime}$ bounds a compressing disk in $H_{b_{1}(\Sigma)}^{\prime}$. Since the splitting (4) is induced by the open book decomposition $(B, \pi)$, we can choose an identification $H_{b_{1}(\Sigma)}^{\prime}=\Sigma \times[0,1]$ such that each $\partial D_{i}^{\prime}$ is of the form

$$
a_{i} \times\{0\} \bigcup \varphi\left(a_{i}\right) \times\{1\},
$$

where $\varphi$ is the monodromy of $(B, \pi)$. The fact that $\partial D_{i}^{\prime}$ bounds a disk in $H_{b_{1}(\Sigma)}^{\prime}$ says that there is a family of arcs in $\Sigma \times I$ interpolating between $a_{i} \times\{0\}$ and $\varphi\left(a_{i}\right) \times\{1\}$. Mapping such family to $\Sigma$ via the projection $\Sigma \times I \rightarrow \Sigma$ shows that each $a_{i}$ is homotopic to $\varphi\left(a_{i}\right)$ (with fixed endpoints), and therefore by [4] each $a_{i}$ is isotopic to $\varphi\left(a_{i}\right)$ via an isotopy which keeps the endpoints fixed. Since $\left\{a_{i}\right\}$ is a system of arcs for $\Sigma$, a standard argument based on the Alexander lemma [5, Lemma 2.1] implies that $\varphi$ is isotopic to the identity of $\Sigma$ via diffeomorphisms which fix $\partial \Sigma$ pointwise. This concludes the proof of Theorem 1.1.

\section{References}

[1] J S Birman, H M Hilden, On isotopies of homeomorphisms of Riemann surfaces, Ann. of Math. 97 (1973) 424-439 MR0325959 
[2] J S Birman, W W Menasco, Studying links via closed braids, V: The unlink, Trans. Amer. Math. Soc. 329 (1992) 585-606 MR1030509

[3] T D Cochran, Non-trivial links and plats with trivial Gassner matrices, Math. Proc. Cambridge Philos. Soc. 119 (1996) 43-53 MR1356156

[4] D B A Epstein, Curves on 2-manifolds and isotopies, Acta Math. 115 (1966) 83-107 MR0214087

[5] B Farb, D Margalit, A primer on mapping class groups, Princeton Mathematical Series 49, Princeton Univ. Press (2012) MR2850125

[6] E Giroux, N Goodman, On the stable equivalence of open books in three-manifolds, Geom. Topol. 10 (2006) 97-114 MR2207791

[7] J E Grigsby, S M Wehrli, An elementary fact about unlinked braid closures arXiv: 1309.0759

[8] W Haken, Some results on surfaces in 3-manifolds, from: "Studies in Modern Topology", Math. Assoc. Amer., Washington, DC (1968) 39-98 MR0224071

[9] A Hatcher, Notes on basic 3-manifold topology (2007) Available at http:// www . math . cornell . edu/ hatcher/3M/3Mfds . pdf

[10] J Hempel, 3-manifolds, Ann. Math. Studies 86, Princeton Univ. Press (1976)

[11] J Johnson, Notes on Heegaard splittings (2006) preprint Available at http:// users.math. yale.edu/ jj327/notes.pdf

[12] Y Ni, Homological actions on sutured Floer homology, Math. Res. Lett. 21 (2014) 1177-1197 MR3294567

[13] L Paris, D Rolfsen, Geometric subgroups of mapping class groups, J. Reine Angew. Math. 521 (2000) 47-83 MR1752295

[14] K Reidemeister, Zur dreidimensionalen Topologie, Abh. Math. Sem. Univ. Hamburg 9 (1933) 189-194 MR3069596

[15] J Singer, Three-dimensional manifolds and their Heegaard diagrams, Trans. Amer. Math. Soc. 35 (1933) 88-111 MR1501673

Laboratoire de Mathématiques Jean Leray, Université de Nantes \& CNRS BP 92208, 2 rue de la Houssinière, F-44322 Nantes 03, France

Dipartimento di Matematica, Università di Pisa

Largo Bruno Pontecorvo 5, I-56127 Pisa, Italy

paolo.ghiggini@univ-nantes.fr, lisca@dm.unipi.it

Received: 18 November 2014 
